\title{
Latent Vector Recovery of Audio GANs with Application in Deepfake Audio Detection
}

\author{
Nicky Bayat ${ }^{\dagger}$, Vahid Reza Khazaie ${ }^{\dagger}$, Andrew Keyes ${ }^{\dagger}$, Yalda Mohsenzadeh †,* \\ $\dagger$ University of Western Ontario \\ London, ON, Canada
}

\begin{abstract}
Advanced Generative Adversarial Networks (GANs) are remarkable in generating intelligible audio from a random latent vector. In this paper, we examine the task of recovering the latent vector of both synthesized and real audio. We propose an autoencoder inspired technique to train a deep residual neural network architecture to project audio synthesized by WaveGAN into the corresponding latent space with near identical reconstruction performance. To accommodate for the lack of an original latent vector for real audio, we optimize the residual network on a multi-level feature loss between the real audio samples and the reconstructed audio of the predicted latent vectors. In the case of synthesized audio, the Mean Squared Error (MSE) between the ground truth and recovered latent vector is minimized as well. We further investigated the audio reconstruction performance when several gradient optimization steps are applied to the predicted latent vector. Through our auto-encoder inspired method of training on real and synthesized audio, we are able to predict a latent vector that corresponds to a reasonable reconstruction of real audio. After training, we investigated the latent representations of real and synthesized audio files. Our analysis revealed distinct latent representational patterns for real and synthesized audio which can be used for deepfake audio detection. Even though we evaluated our method on WaveGAN, our proposed method is universal and can be applied to any other GANs.
\end{abstract}

\section{INTRODUCTION}

Researchers have recently shown an increased interest in mapping generated samples by generative adversarial networks (GANs) into the original latent vectors. GANs consist of two major components: the generator and the discriminator. The generator receives a random latent vector and generates realistic samples through a min-max optimization game with the discriminator. The discriminator aims to determine whether a sample is real or fake. The problem of GAN inversion refers to the task of recovering a latent vector for a given sample (image, audio, video, etc.) that when given to the generator, it can precisely regenerate the target. Projecting generated samples back to the latent-space is a classic problem in computer vision, where the goal is to recover the latent vector of given images. However, investigating the inverse mapping of GANs is still a continuing concern within the audio domain. While methods introduced in the vision domain can be applied to audio as well, they need to be improved and fine-tuned to this domain in order to produce ideal results.

Recent advances in the inverse mapping of adversarial generators, in particular inverse mapping of image generators [1-4], have led to the question of whether it is possible to map audio to a latent vector that can regenerate it. Three major solutions have been proposed for projecting images back to the latent vector peers. There is a large and growing body of the literature that recovers the latent vector through an optimization-based approach [1]. This technique updates an initial random z vector using gradient descent until the loss between the sample generated by this vector and the target is less than a desired threshold. While this method can recover accurate latent vectors, it often takes too long to find the solution. Auto-encoder inspired architectures are the second most common solution to this 


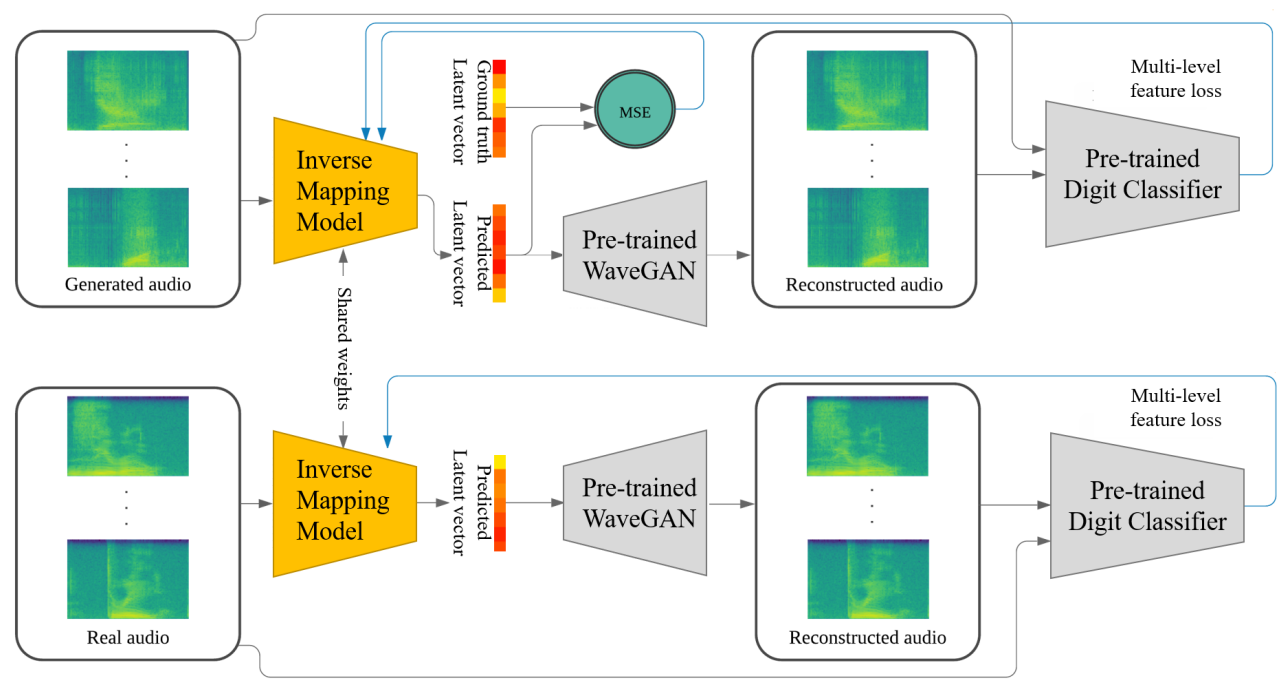

Figure 1. Our proposed framework for training an Inverse Mapping Model for Real Audio. During each epoch, the model was first trained on one batch of real audio with multi-level feature loss and then one batch of WaveGAN synthesized audio with a combination of MSE between the original and predicted latent vectors and multi-level feature loss between the original audio and the corresponding reconstructions.

problem [5][6][7][3]. In this method, an encoder network is trained to map generated samples to latent vectors. Next, the generator (decoder) uses these vectors to regenerate the target sample. Hybrid methods are another approach that benefits from the advantages of both mentioned techniques [4]. They first predict an initial latent vector via a deep network and later update via a few further steps of gradient descent.

It has been previously observed that it is possible to map synthesized images to latent vectors that regenerate indistinguishable results from the target. The main challenge faced by many researchers is the lack of ground truth latent vectors for real samples. In this paper, we propose an auto-encoder inspired method that trains a residual neural network encoder model (ResNet-18 [8]) to predict latent vectors of audio samples (synthesized or real). Our inverse mapping model is trained based on a combination of MSE and multi-level feature loss. Despite its many applications, there has been no detailed investigation on recovering the latent vectors of audio. This paper attempts to show that common approaches used for projecting images to latent-space can be used on audio, but they need to be fine-tuned and adjusted for this task. To the best of our knowledge, the experimental work presented here provides one of the first investigations into how to project audio samples to latent representations that can regenerate them. In this paper our contributions are: (1) We propose a fast auto-encoder inspired technique to recover latent vectors of both fake and real audio. (2) We propose a novel multi-level feature loss within the audio domain. (3) We also perform latent vector recovery using gradient descent methods in the audio domain. (4) We implement a hybrid method that applies a few steps of gradient descent after predicting the representation by the encoder network and compare the results with the proposed autoencoder inspired framework and sole gradient descent approach. (5) We show that our method results in distinct latent representational patterns for real and synthesized audio that can be used for deepfake audio detection. 


\section{METHOD}

A major advantage of using auto-encoder inspired networks is that they are faster compared to gradient-based alternatives [3]. In this work, we trained a ResNet-18 model on a dataset of generated audio and their corresponding ground truth latent vector to learn the inverse mapping. To the best of our knowledge, we are the first to employ a multi-level feature loss besides the MSE loss in training an audio inverse mapping model. WaveGAN was the first attempt at applying GANs to unsupervised synthesis of raw-waveform audio [9]. The model is capable of producing one second clips at a frequency of $16 \mathrm{khz}$ learned from a variety of audio datasets. We chose to use the official WaveGAN model pre-trained on the SC09 (subset of Speech Commands) dataset [10] in this work.

Our goal for the inverse mapping model is to take as input the spectrogram of generated and real audio and output the predicted latent vector for a pre-trained WaveGAN. The inverse mapping model architecture is the same as ResNet-18 with the last layer removed and a new layer added with the size of the WaveGAN latent space. At each epoch, the inverse mapping model was trained one batch on audio synthesized by WaveGAN and the next batch on real audio from the SC09 dataset. The architecture of our proposed method is presented in Figure 1. At run time, audio was synthesized by WaveGAN from a random latent vector with a uniform distribution between -1 and 1 . In the case of synthesized audio, two losses were used to train the network. The multi-level feature loss and the MSE between the original latent vector (used as input to the WaveGAN) and the predicted latent vector (by the inverse mapping model). For real audio, only the multi-level feature loss was used as we do not have a true latent vector. In sum, to train the inverse mapping model, we employed two types of objective functions: (1) MSE between latent vectors: With the synthesized audio as input to the inverse mapping model, the latent vector was predicted in the last layer of the network. The ground truth latent vector was then compared to the predicted latent vector through mean squared error. This loss is necessary to learn the inverse mapping between the audio and latent vectors. (2) Multi-level feature loss: The multi-level feature loss was quantified as the difference (MSE) in activations of a ResNet-18 digit classifier's output at each residual block. The classifier was pre-trained on the SC09 dataset. This loss compares the audio feature representations at multiple level and could improve the reconstruction of real audio in style and content.

\section{EXPERIMENTS AND RESULTS}

In this section, we compare the inverse mapping model with sole gradient descent and hybrid methods based on Inception Score (IS), Frechet Inception Distance (FID), MSE distance, the Structural Similarity Index measure (SSIM) and digit classification accuracy on both real and synthesized audio reconstruction.

\subsection{Latent Vector Recovery with Gradient Descent}

In order to recover the latent representation of a given audio using this method, we first sample a random vector, $z^{*}$, from the uniform distribution. Then we feed this latent vector to WaveGAN to get the corresponding audio. Next, we convert the audio to a spectrogram and compute the Mean Absolute Error (MAE) loss between the generated spectrogram and the target spectrogram. Afterward, $z^{*}$ is updated by the SciPy optimize library using the LBFGS algorithm [11]. After each iteration, the updated $z^{*}$ is closer to the ground truth latent vector. The main disadvantage of this method is that it is very slow. Numerous iterations are required to find the optimum latent vector for a target sample; further, finding the perfect hyperparameters (learning rate and number of iterations) is extremely challenging. 


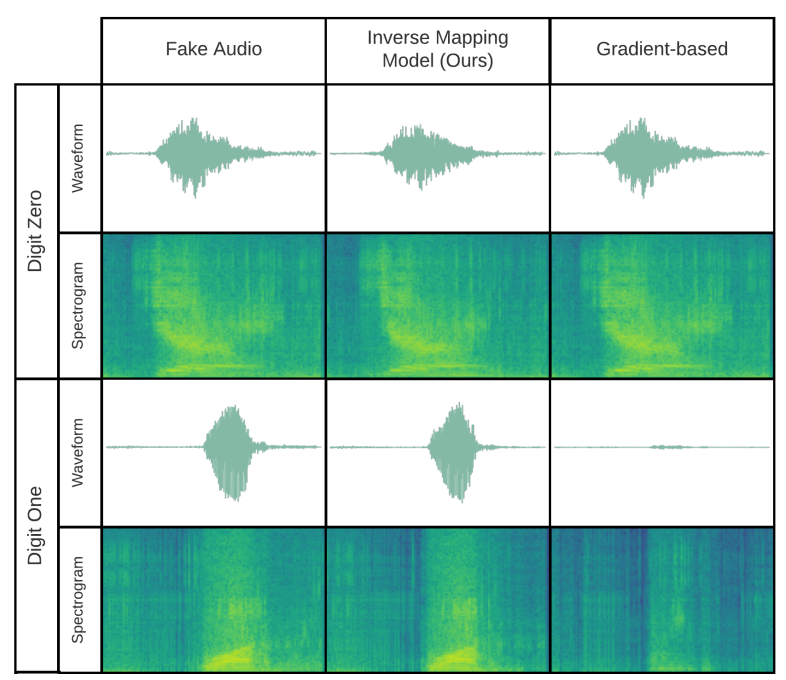

Figure 2. Comparing the performance of our inverse mapping model with gradient descent approach in latent vector recovery of synthesized audio for two example digits.

\subsection{Experiment 1: Recovering Latent Vectors for Synthesized Audio Reconstruction}

The purpose of Experiment 1 was to evaluate the performance of inverse mapping model trained using MSE and multi-level feature loss in latent vector recovery of synthesized audio. We implemented an optimization-based method that updates a random vector for a maximum of 50000 steps using gradient descent. In addition, we developed a hybrid method that first predicts a latent vector using our inverse mapping model and then updates it further with a maximum of 200 steps of gradient descent. The results in Table 1 show that the recovery through our inverse mapping model produces a more coherent spoken digit with better content and style reconstruction. The qualitative results of our approach on fake audio are shown in Figure 2 where our method is compared to gradient optimization for recovering the raw waveform and spectrogram of the audio.

Table 1. Performance Comparison in Synthesized Audio Reconstructions. Higher Inception Score (IS) indicates better auditory quality. The lower FID score indicates better performance with 0 indicating the two latent vectors are identical. MSE measures the mean squared error between latent vectors, the lower the error, the better. SSIM measures the similarity between spectrograms, a higher value indicates more similarity.

\begin{tabular}{|c|c|c|c|c|}
\hline & $\begin{array}{c}\text { Inception Score } \\
(\text { mean } \pm \text { std) }\end{array}$ & FID & $\begin{array}{c}\text { MSE } \\
\text { (raw audio) }\end{array}$ & $\begin{array}{c}\text { SSIM } \\
\text { (spectrogram) }\end{array}$ \\
\hline Fake & $7.23 \pm 0.003$ & - & - & - \\
\hline Gradient-based & $3.96 \pm 0.203$ & 0.7140 & 0.00489 & 0.9618 \\
\hline Inverse Mapping Model (ours) & $7.50 \pm 0.288$ & 0.4841 & 0.00196 & 0.9731 \\
\hline Hybrid Method (ours) & $7.23 \pm 0.006$ & 0.4850 & 0.00003 & 0.9979 \\
\hline
\end{tabular}

\subsection{Experiment 2: Recovering Latent Vectors for Real Audio Reconstruction}

A major problem with mapping real audio is that there are no ground truth latent vectors that accurately generate them. Another issue is the limitation of the audio GANs themselves, they are not capable of generating high quality naturalist audio that sound identical to the real peers. Performance comparison of our inverse mapping model with the 


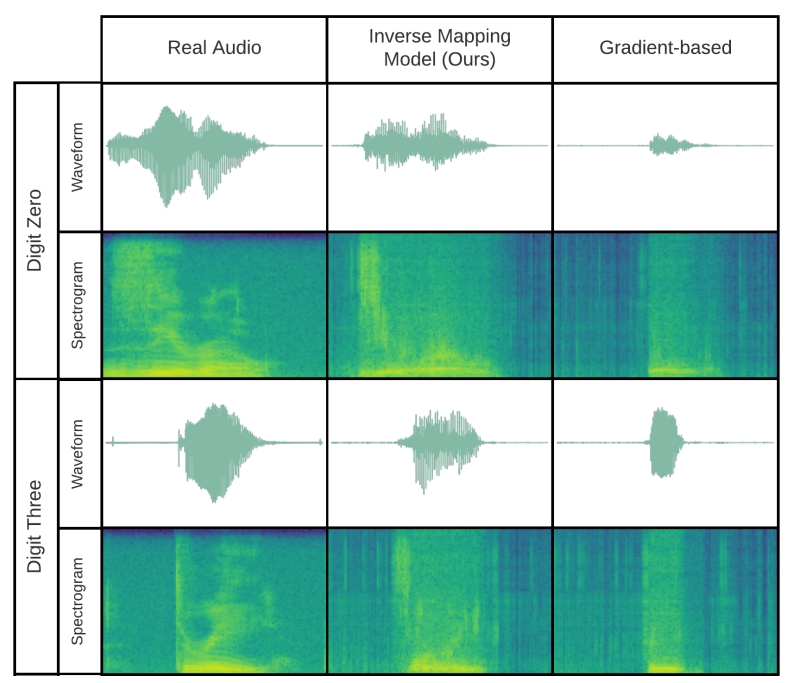

Figure 3. Comparing the performance of our inverse mapping model with gradient descent approach in latent vector recovery of real audio for two example digits.

Table 2. Performance Comparison in Real Audio Reconstructions

\begin{tabular}{|c|c|c|c|c|c|}
\hline & $\begin{array}{c}\text { Inception Score } \\
(\text { mean } \pm \text { std) }\end{array}$ & FID & $\begin{array}{c}\text { MSE } \\
\text { (raw audio) }\end{array}$ & $\begin{array}{c}\text { SSIM } \\
\text { (spectrogram) }\end{array}$ & Accuracy \\
\hline Real & $9.20 \pm 0.019$ & - & - & - & $95.41 \%$ \\
\hline Gradient-based & $2.20 \pm 0.063$ & 1.0566 & 0.00959 & 0.953 & $17.17 \%$ \\
\hline Inverse Mapping Model (ours) & $7.93 \pm 0.077$ & 0.4514 & 0.01176 & 0.946 & $71.06 \%$ \\
\hline Hybrid Method (ours) & $3.89 \pm 0.027$ & 0.8976 & 0.00887 & 0.954 & $36.93 \%$ \\
\hline
\end{tabular}

alternatives on projecting real audio to latent representations is summarized in Table 2. While gradient-based methods reconstruct the waveform and spectrograms well, in terms of classification accuracy, our inverse mapping model is significantly better and is closest to the real audio accuracy. Qualitative comparison on real audio is presented in Figure 3.

\subsection{Experiment 3: Separability in the Latent Space with Application in Deepfake Detection}

To further investigate the characteristics of the predicted latent vectors, we designed a deepfake detection experiment to see whether our inverse mapping model can be applied to this task. For this purpose, we randomly selected 100 samples from SC09 dataset and 100 fake synthesized audio by WaveGAN. We first trained and tested two binary classifiers with 10-fold cross validation procedure on the audio spectrograms to perform real and fake discrimination. Then, we mapped the audio to the latent space with our inverse mapping model and trained and tested the same two binary classifiers on the predicted latent vectors for real/fake discrimination. The results are reported in Table 3. As depicted using latent vectors significantly improved the classification performance of both Logistic Regression and SVM classifier. Finally, we used t-SNE method and visualized real and fake audio data in 2 dimensions in Figure 4A and the latent vectors of real and fake audio in Figure 4B. As depicted our inverse mapping model clearly separates real and fake audio in the latent space. 

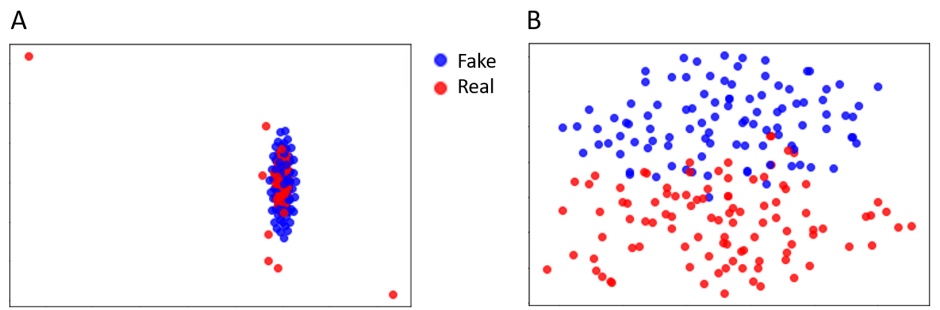

Figure 4. 2D t-SNE visualization of (A) audio spectrograms and (B) predicted latent vectors.

Table 3. Deepfake detection using audio spectrograms versus predicted latent vectors

\begin{tabular}{|c|c|c|}
\hline & Logistic Regression (\%) & SVM (\%) \\
\hline Spectrograms & 51.5 & 67.49 \\
\hline Predicted Latent Vectors & 91.99 & 91.5 \\
\hline
\end{tabular}

\section{CONCLUSION}

The present research aimed to examine different approaches of latent vector recovery for audio GANs. We proposed an auto-encoder inspired approach to project both synthesized and real audio into latent space. We trained a ResNet-18 architecture based on a combination of MSE and multi-level feature losses. Deep network encoder solutions are faster than optimization-based alternatives since they do not require multiple iterations of optimization. In this study, we demonstrated that deep neural network encoders are also more accurate in terms of both auditory quality of the reconstructed sounds and digit classification accuracy. We further showed that our proposed model maps real and synthesized audio to separable latent space which can be used for applications like deepfake detection.

\section{References}

[1] A. Creswell and A. A. Bharath. "Inverting the generator of a generative adversarial network". In: IEEE transactions on neural networks and learning systems 30.7 (2018), pp. 1967-1974.

[2] Z. C. Lipton and S. Tripathi. "Precise recovery of latent vectors from generative adversarial networks". In: arXiv preprint arXiv:1702.04782 (2017).

[3] N. Bayat, V. R. Khazaie, and Y. Mohsenzadeh. Inverse mapping of face GANs. 2020. arXiv: 2009.05671 [cs.CV].

[4] J.-Y. Zhu, P. Krähenbühl, E. Shechtman, and A. A. Efros. "Generative visual manipulation on the natural image manifold". In: European conference on computer vision. Springer. 2016, pp. 597-613.

[5] A. Van Den Oord, O. Vinyals, et al. "Neural discrete representation learning". In: Advances in Neural Information Processing Systems. 2017, pp. 6306-6315.

[6] J. Donahue, P. Krähenbühl, and T. Darrell. "Adversarial feature learning". In: arXiv preprint arXiv:1605.09782 (2016).

[7] V. Dumoulin, I. Belghazi, B. Poole, O. Mastropietro, A. Lamb, M. Arjovsky, and A. Courville. "Adversarially learned inference". In: arXiv preprint arXiv:1606.00704 (2016).

[8] K. He, X. Zhang, S. Ren, and J. Sun. "Deep residual learning for image recognition". In: Proceedings of the IEEE conference on computer vision and pattern recognition. 2016, pp. 770778.

[9] C. Donahue, J. J. McAuley, and M. S. Puckette. "Synthesizing Audio with Generative Adversarial Networks". In: CoRR abs/1802.04208 (2018). arXiv: 1802.04208.

[10] P. Warden. "Speech commands: A dataset for limited-vocabulary speech recognition". In: arXiv preprint arXiv:1804.03209 (2018).

[11] D. C. Liu and J. Nocedal. "On the limited memory BFGS method for large scale optimization". In: Mathematical programming 45.1 (1989), pp. 503-528. 